\title{
Excretion of anthranilate and 3-hydroxyanthranilate by Saccharomyces cerevisiae: relationship to iron metabolism
}

\author{
E. Lesuisse, ${ }^{1}$ M. Simon, ${ }^{1}$ R. KLein ${ }^{2}$ and P. LABbe ${ }^{1 *}$ \\ ${ }^{1}$ Laboratoire de Biochimie des Porphyrines, Institut J. Monod, Tour 43, Université Paris 7, 2 Place Jussieu, \\ 75251 Paris cedex 05, France \\ ${ }^{2}$ Laboratoire de Physique et Chimie Biomoléculaire, CNRS UA 198, Institut Curie, 11 rue P. et M. Curie, \\ 75231 Paris cedex 05, France
}

(Received 29 July 1991; revised 17 September 1991; accepted 1 October 1991)

\begin{abstract}
Resting suspensions of cells of Saccharomyces cerevisiae grown in iron-rich or iron-deficient conditions were studied by following the fluorescence emission changes $\left(\lambda_{\text {em. }} .400-460 \mathrm{~nm}, \lambda_{\text {exc. }} 300-340 \mathrm{~nm}\right)$ occurring in these suspensions upon addition of glucose and ferric iron. The results show that, in addition to NAD(P)H, metabolites of the aromatic amino acid pathway interfere with the fluorescence measurements, and that they could be involved in ferric iron reduction. Wild-type strains of $S$. cerevisiae are known to excrete anthranilic acid and 3hydroxyanthranilic acid in response to glucose. The major fluorescing compound excreted by a chorismatemutase-deficient mutant strain of $S$. cerevisiae was identified as anthranilic acid. The excretion of anthranilic and 3-hydroxyanthranilic acids was correlated with the ferric-reducing capacity of the extracellular medium. Excretion during growth was much greater by cells cultured in iron-rich medium than by cells grown in iron-deficient medium. The possibility was examined that a link could exist between the biosynthesis of aromatics and the ferri-reductase activity of the cells, via chorismate synthase and its putative diaphorase-associated activity. Two ferri-reductasedeficient mutants excreted much less 3-hydroxyanthranilate than did the parental wild-type strains. However, the ferri-reductase activity of a chorismate-synthase-deficient mutant was comparable to that of the parental strain.
\end{abstract}

\section{Introduction}

Many micro-organisms respond to iron limitation by excreting low molecular mass siderophores. These are hydroxamates or catecholates that chelate ferric iron, making it available for transport (Winkelmann \& Huschka, 1987). However, the yeast Saccharomyces cerevisiae excretes no siderophores. The main strategy used by this organism for iron acquisition is similar to that of many plants: a trans-plasma-membrane redox system catalyses electron transfer from the interior of the cells to extracellular ferric chelates. The $\mathrm{Fe}^{2+}$ ions are then taken up by the cells independently (Lesuisse $e t$ al., 1987; Lesuisse \& Labbe, 1989; Dancis et al., 1990).

Several plants and micro-organisms, when grown in iron-limited conditions, also release fluorescing molecules, very often small phenolic compounds. Those molecules are not siderophores but they could facilitate the transport of iron into the cell in several ways: they

\footnotetext{
* Author for correspondence. Tel. (1) 435404 79; fax (1) 46332305 .

Abbreviations: AA, anthranilic acid; OH-AA, 3-hydroxyanthranilic acid.
}

could maintain $\mathrm{Fe}^{2+}$ in the reduced state after reduction of the ferric species at the plasma membrane and/or they could solubilize iron in the growth medium or even directly reduce certain ferric species (Olsen et al., 1981; Romheld, 1987). Such reducing/chelating compounds were previously shown to be excreted by $S$. cerevisiae, but they were not identified (Lesuisse et al., 1987).

The present paper re-examines the production and excretion of fluorescing material by $S$. cerevisiae in relation to the iron status of the cells.

\section{Methods}

Yeast strains, growth conditions and ferri-reductase activity of the cells. Mutant strains affected in certain steps of the aromatic amino acid biosynthetic pathway were obtained from the collection of $\mathrm{G}$. H. Braus (ETH, Mikrobiologisches Institut, Zürich, Switzerland): RH1127 (a chorismate-synthase-deficient mutant), RH1242 (a chorismate-mutase-deficient mutant), RH712 (an anthranilate-synthase-deficient mutant) and X2180-1A (a parental strain of RH1127, RH1242 and RH712). W103 is a ferri-reductase-deficient mutant (Dancis et al., 1990) and F113 a parental strain of W103; G204 is an haem-deficient 
mutant which is also deficient in ferri-reductase activity (Lesuisse \& Labbe, 1989), and FL200 is a parental strain of G204.

The cells were precultured and cultured either in complete media (containing about $10 \mu \mathrm{M}$-iron) or in iron-rich $[180 \mu \mathrm{M}$-Fe(III)-citrate] or iron-deficient (less than $10 \mathrm{~nm}$ residual iron) media as previously described (Lesuisse \& Labbe, 1989). The iron-trapping reagent ferrozine $\left(50 \mathrm{mg}^{-1}\right)$ was added to iron-deficient media to decrease the availability of residual iron. The ferri-reductase activity of the washed cells was determined spectrophotometrically (Lesuisse et al., 1987), with $360 \mu \mathrm{M} \mathrm{Fe}(\mathrm{III})$-citrate as substrate and $5 \%(\mathrm{w} / \mathrm{v})$ glucose in $50 \mathrm{~mm}$ citrate buffer, $\mathrm{pH}$ 6.5.

For measuring the correlation between the ferric-reducing capacity of the extracellular medium and excretion by the cells of 3hydroxyanthranilic acid (OH-AA) or anthranilic acid (AA), cells grown in either iron-rich or iron-deficient medium were washed and suspended at $20 \mathrm{mg}^{\text {wet }} \mathrm{wt} \mathrm{ml}^{-1}$ in $50 \mathrm{~mm}-\mathrm{MOPS}$ buffer, $\mathrm{pH}$ 7. The cell suspension was incubated at $30^{\circ} \mathrm{C}$ in the presence of $5 \%(w / v)$ glucose. Samples $(1 \mathrm{ml})$ were withdrawn at intervals (about every $10 \mathrm{~min}$ ), centrifuged, and the supernatants were divided into two portions. To one portion, $360 \mu \mathrm{M}$-Fe(III)-citrate and $1 \mathrm{~mm}$-bathophenanthroline sulphonate (BPS) was added; after 15 min incubation in the dark the amount of $\mathrm{Fe}(\mathrm{II})$ was determined spectrophotometrically $\left[\varepsilon_{(\mathrm{BPS})_{3}-\mathrm{Fe}(\mathrm{II})}\right.$ $\left.=19.5 \mathrm{~mm}^{-1} \mathrm{~cm}^{-1}\right]$. The second portion of each supernatant was used to assay AA and OH-AA fluorimetrically as described below.

Kinetics of fluorescence changes in suspensions of whole cells. The kinetics were followed in a Jobin-Yvon JY3D thermostated recording spectrofluorimeter equipped with a Hamamatsu R928H4 photomultiplier tube. Despite the theoretical limitations imposed by the high turbidity of the yeast cell suspensions when using a classical fluorimeter, we found a good relationship between fluorescence intensity and the number of yeast cells for the Jobin-Yvon spectrofluorimeter, approaching that measured in the Eppendorf photometer 1101 $M$ with fluorimeter attachment (Estabrook, 1962). The cells (20 mg wet wt $\left.\mathrm{ml}^{-1}\right)$ were incubated in MOPS buffer $(50 \mathrm{mM}, \mathrm{pH} 7)$ at $30^{\circ} \mathrm{C}$ with magnetic stirring in a $3 \mathrm{ml}$ quartz cuvette with an excitation beam $\left(\lambda_{\text {exc. }}\right)$ of $300-340 \mathrm{~nm}$, and the fluorescence emission $(400-460 \mathrm{~nm})$ was recorded as a function of time. The band widths of the four slits were: 2 $\mathrm{nm}$ each for the excitation monochromator entry and exit and $4 \mathrm{~nm}$ for the emission monochromator entry and $20 \mathrm{~nm}$ for the exit. Excitation and/or emission spectra were recorded at specific times during incubation in some of the assays.

Measurements of $A A, O H-A A$ and tryptophan in whole cells, and in the culture and/or incubation medium. AA and $\mathrm{OH}-\mathrm{AA}$ concentrations in iron-rich and iron-deficient cultures were determined fluorimetrically after extracting cell-free samples with ethyl acetate (Miozzari et al., 1978).

Intracellular AA and OH-AA were determined fluorimetrically in cell extracts prepared by incubating cells in hot $\left(90^{\circ} \mathrm{C}\right)$ water at $100 \mathrm{mg}$ wet $\mathrm{wt} \mathrm{ml}^{-1}$ for $20 \mathrm{~min}$.

The fluorescent molecules (including tryptophan, AA and OH-AA) excreted by the cells in resting suspensions were assayed directly in the supernatants from centrifuged cell suspensions.

In each case, the excitation spectrum was recorded and the amounts of AA or OH-AA and eventually of tryptophan were determined by reference to standard curves obtained with commercial products (AA from Fluka, OH-AA and tryptophan from Aldrich).

\section{Results and Discussion}

\section{Excretion of aromatics by cells}

These studies were carried out to estimate the relationship between ferric iron reduction by whole cells

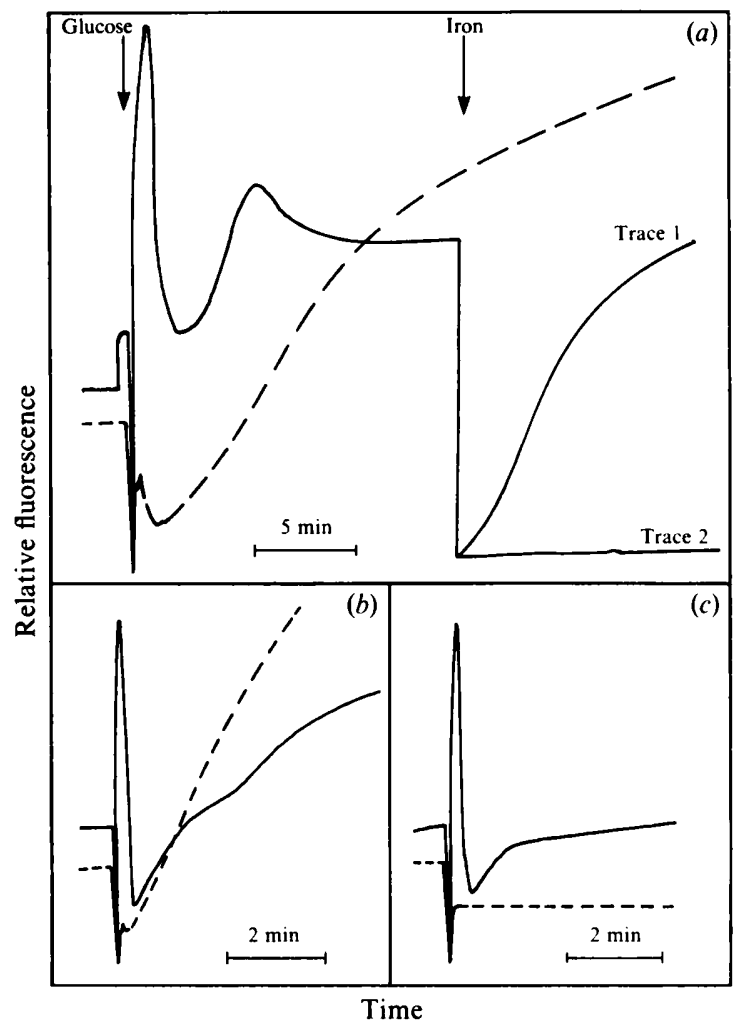

Fig. 1. Kinetics of fluorescence changes in resting suspensions of cells of different strains. The emission fluorescence changes were followed at two pairs of wavelengths: (i) $\lambda_{\text {exc. }}=340 \mathrm{~nm}, \lambda_{\text {em. }}=450 \mathrm{~nm}$ (solid lines) and (ii) $\lambda_{\text {exc. }}=325 \mathrm{~nm}, \lambda_{\text {em. }}=400 \mathrm{~nm}$ (dashed lines). Cells were incubated as described in Methods. At the time indicated by the first arrow, glucose was added to a final concentration of $5 \%(w / v)$. The second arrow in (a) indicates the addition of iron [as $360 \mu \mathrm{M}$-Fe(III)citrate]. Cells of strain Fl200 (a) were grown for $6 \mathrm{~h}$ in iron-rich (trace 2) or iron-deficient medium (trace 1). Cells of strains RH1242 $(b)$ and RH712 (c) were grown for $15 \mathrm{~h}$ in complete medium and harvested in the experimental phase. Cells in $(b)$ and $(c)$ were treated as in $(a)$ except that no iron was added.

and the change in intracellular $\mathrm{NAD}(\mathrm{P}) \mathrm{H}$, monitored according to Estabrook (1962). Resting suspensions of intact yeast cells grown under iron-rich or iron-deficient conditions were used to follow the changes in fluorescence emission ( $\lambda_{\text {exc. }} 300-340 \mathrm{~nm}, \lambda_{\text {em. }} 400-460 \mathrm{~nm}$ ) occuring upon addition of substrates, ferric salts and/or inhibitors. Our recordings were highly reproductible (Fig. 1). The changes in fluorescence emission at $450 \mathrm{~nm}$ with $\lambda_{\text {exc. }}=340 \mathrm{~nm}$ essentially reflect the changes in intracellular levels of reduced pyridine nucleotides and in the rate of NADH binding, since bound NADH fluoresces 8-12-fold more than free NADH (Estabrook. 1962). An initial increase occurred when the cell suspension reached anaerobiosis and there was a peak of fluorescence immediately after adding glucose; these changes were similar in all the strains tested (Fig. $1 a-c$ ), for both iron-rich and iron-deficient cells (Fig. $1 a$ ). The 


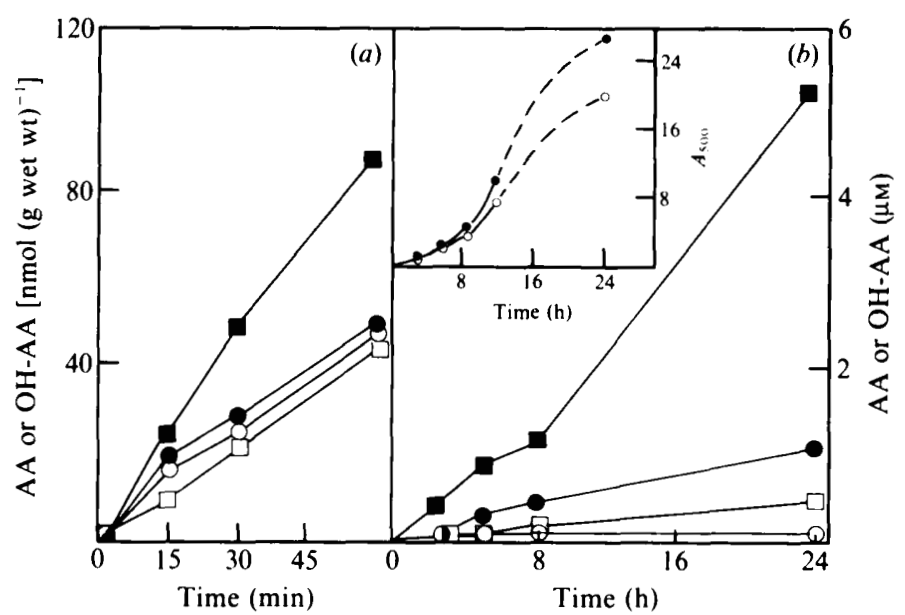

\begin{abstract}
Fig. 2. Time-course of $\mathrm{AA}$ and $\mathrm{OH}-\mathrm{AA}$ excretion by iron-deficient and iron-rich cells in resting conditions (a) or by growing cells in iron-rich or iron-deficient medium $(b)$. Samples were withdrawn and centrifuged at the times indicated; AA (strain RH1242) and OH-AA (strain X2180-1A) were assayed in the supernatants as described in Methods. $\square, \square$, Strain RH1242; O, O, strain X2180-1A; open symbols, iron-deficient conditions; filled symbols, iron-rich conditions. Insert: growth of strain X2180-1A in iron-rich $(O)$ or iron-deficient $(O)$ media.
\end{abstract}

addition of a ferric salt [Fe(III)-citrate, Fe(III)-EDTA, ferricyanide] to the cell suspensions resulted in a sudden drop in fluorescence emission which was followed by a new increase in the case of iron-deficient cells only (Fig. $1 a)$. The increase in fluorescence emission was maximum at $\lambda_{\text {em. }}=415 \mathrm{~nm}\left(\lambda_{\text {exc. }}=325 \mathrm{~nm}\right)$ for a wild-type strain (FL200 or X2180-1A) and at $\lambda_{\text {em. }}=400 \mathrm{~nm}\left(\lambda_{\text {exc. }}\right.$. $=318 \mathrm{~nm}$ ) for a strain deficient in chorismate mutase activity (RH1242). The rate of increase was closely related to the ferri-reductase activity of the cells: with all strains tested and all substrates used [ferricyanide, $\mathrm{Fe}$ (III)-EDTA, Fe(III)-citrate], the rate of fluorescence increase after ferric iron addition was always strictly proportional to the rate of iron reduction by the cells, whatever their growth conditions, and both processes were inhibited at identical rates by the same inhibitors (diethylstilboestrol, vanadate, permeabilizing agents, uncouplers; data not shown). The typical recording in Fig. $1(a)$ results from the following changes. First, the drop in fluorescence emission after addition of ferric iron is essentially due to the ferric salts quenching the fluorescence of all molecules absorbing in the UV (up to $400 \mathrm{~nm}$ ), including of course NAD(P)H. [The drop was proportional to the absorbance, in the range $330-340 \mathrm{~nm}$, of the reagent added: $\mathrm{Fe}(\mathrm{III})$-citrate $>\mathrm{Fe}$ (III)-EDTA $>$ ferricyanide; no effect of $\mathrm{Fe}^{2+}$ or of other cations such as $\mathrm{Cu}^{2+}, \mathrm{Ga}^{3+}, \mathrm{Al}^{3+}, \mathrm{Cr}^{3+}, \mathrm{Co}^{2+}$. The subsequent increase in fluorescence emission that occurs in irondeficient cell suspensions is mainly due to a fluorescing compound partly excreted into the extracellular medium and results from the decrease of the quenching effect of ferric iron which is rapidly reduced by the cells with induced ferri-reductase activity (iron-deficient cells) into non-quenching ferrous iron. The excretion of a fluorescent molecule [maximum excitation at $325 \mathrm{~nm}$ (FL200) or at $318 \mathrm{~nm}$ (RH1242)] by the resting cells was monitored by following the excitation spectra $\left(\lambda_{\text {exc. }}=\right.$ $300-360 \mathrm{~nm}, \lambda_{\text {em. }}=400$ or $\left.415 \mathrm{~nm}\right)$ of centrifuged samples (data not shown). The total production of these fluorescent compounds by the resting cells was also followed continuously (Fig. $1 a-c$, dashed lines); it was stimulated by adding glucose (Fig. $1 a, b$ ) and did not occur in a strain deficient in anthranilate synthase activity (Fig. 1c). The intensity of extracellular fluorescence emission was proportional to the ferric reducing capacity of the extracellular medium (data not shown). We have previously shown that one or more reducing compound(s) is/are excreted by yeast cells incubated with glucose, but the molecule(s) was not identified (Lesuisse et al., 1987). HPLC analysis (unpublished results) and comparisons with the fluorescence properties of commercial products indicate that the major fluorescent compound excreted by the mutant strain RH1242 is anthranilic acid, and the major fluorescent compound excreted by the wild-type strains is 3hydroxyanthranilic acid. To our knowledge, this is the first time that $\mathrm{OH}-\mathrm{AA}$ acid has been detected in $S$. cerevisiae.

\section{Relationships with iron metabolism}

Small molecules derived from the metabolism of aromatic amino acids and having iron-reducing/chelating properties are known to be excreted by several plants and micro-organisms in relation to their iron status [e.g. excretion of caffeic acid by plants (Römheld, 1987), excretion of 2, 3 dihydroxybenzoate (Downer et al., 1970) or anthranilate (Rioux et al., 1986) by bacteria]. To determine whether such an interaction between excretion of aromatics and iron metabolism exists in $S$. cerevisiae, we monitored excretion of AA and OH-AA by cells under two experimental conditions: resting cells previously grown in iron-rich or iron-deficient medium, and growing cells in iron-rich or iron-deficient medium (Fig. 2). Excretion of OH-AA by cells of a wild-type strain (X2180-1A) in resting conditions was independent 
of their iron status (Fig. 2a), confirming our previous report (Lesuisse et al., 1987) that iron-deficient and ironrich cells in resting suspension excrete similar amounts of iron-reducing compounds. In contrast, resting cells of the mutant strain RH1242 (which overproduced AA) that had been grown in iron-rich medium excreted more AA than those that had been grown in iron-deficient medium (Fig. $2 a$ ); in the same conditions, this strain also excreted tryptophan, at a rate [about $20 \mathrm{nmol} \mathrm{h}^{-1}$ (g wet wt) ${ }^{-1}$ ] that was independent of the iron status of the cells (data not shown). Both strains excreted more AA and OH-AA during growth in iron-rich medium than in iron-deficient medium (Fig. 2b). The intracellular levels of AA and $\mathrm{OH}-\mathrm{AA}$ varied little (data not shown). This result was not due to a reduced viability of the cells growing in ironpoor medium, since AA and OH-AA excretion increased significantly as soon as the cells were transferred to ironrich medium, before any difference in growth could be detected (Fig. $2 b$ ). The results in Fig. 2 do not seem to indicate that excretion of AA and $\mathrm{OH}-\mathrm{AA}$ plays a physiological role in iron assimilation by the cells, since excretion did not increase in response to iron-limitation.

\section{Excretion of aromatics and cell ferri-reductase activity}

Gaines et al. (1981) showed that the siderophore reductase activity of Bacillus subtilis is associated with two aromatic biosynthetic enzymes, chorismate synthase and dehydroquinate synthase. They proposed that 'a portion of the ferrisiderophore reductase activity in $B$. subtilis could be catalysed by a reductase that also is essential for aromatic biosynthesis'. Reciprocally, Hasan \& Nester (1978) showed that an NADPH-dependent flavin reductase exhibiting a diaphorase activity was required for activation of chorismate synthase in $B$. subtilis. The purified protein had a low pI (4.75), was specific for NADPH and required FMN for reduction of 2,6-dichlorophenol-indophenol. We have found that the ferri-reductase purified 100-fold from plasma membranes of $S$. cerevisiae has similar characteristics (Lesuisse et al., 1990). The chorismate synthase of the fungus Neurospora crassa is also a flavoprotein, with an intrinsic NADPH-dependent activity, and it was suggested that the diaphorase activity might have importance as a regulatory mechanism (Welch et al., 1974). There could therefore be an interaction between aromatic amino acid biosynthesis and iron metabolism via chorismate synthase in $S$. cerevisiae, involving a multifunctional reductive system that both promotes the reductive assimilation of iron and activates chorismate synthase.

Our experiments favour the existence of a link between aromatic metabolism and the ferri-reductase activity of the cells. Cells grown in iron-rich or in irondeficient medium with tryptophan or phenylalanine as carbon source exhibited a 2-fold greater ferri-reductase activity than did control cells grown with glutamate or glucose (data not shown). In addition, the ferri-reductase activity of whole cells of a histidine auxotroph (strain FL200) was 3-fold higher when the cells were incubated for $2 \mathrm{~h}$ in a minimal medium without amino acids than when they were incubated in the same medium supplemented with all 20 amino acids (the cells were precultured for $24 \mathrm{~h}$ in both cases in the same aminoacid-rich medium). Chorismate synthase activity is similarly activated in $S$. cerevisiae: the corresponding $A R O 2$ gene is regulated by the general control activator protein GCN4 (Jones \& Braus, 1990). Finally, the production of anthranilate by resting cells of strain RH1242 was strongly inhibited by $75 \mu \mathrm{M}$-diethylstilboestrol, which also strongly inhibits the ferri-reductase activity of the cells at this concentration (unpublished results). Limited amounts $(10 \mu \mathrm{M})$ of the artificial electron acceptor resazurin also produced a transient inhibition. Resazurin, which is a low-affinity substrate of the ferri-reductase, was used instead of ferric iron because of its low quenching effect at low concentration. These inhibitory effects were more pronounced with the strain RH1242 than with strains producing OH-AA instead of AA.

The possible link between chorismate synthase and the ferri-reductase activity of the cells was further investigated by using the chorismate-synthase-deficient mutant strain RH1127 and the mutant strains W103 and G204, which are deficient in ferri-reductase activity. As shown in Fig. 3(a), the strains G204 and W103 excreted very little $\mathrm{AA}$ and $\mathrm{OH}-\mathrm{AA}$ compared to their parental strains. However, the chorismate-synthase-deficient strain (RH1127) had normal ferri-reductase activity that was even lower than that of the parental strain (Fig. $3 b$ ).

\section{General conclusions}

Micro-organisms which produce catecholate-type siderophores in response to iron-limitation should be able to regulate carbon flux through their aromatic biosynthetic pathways as a function of the iron status of the cells. The same could be true for the organisms like $S$. cerevisiae, that excrete small aromatic compounds having ferric chelating/reducing properties. A simple regulatory model was proposed for B. subtilis (Gaines et al., 1981): in this organism, a multifunctional reductase acts either as an activator of chorismate synthase - accounting for the increased production of dihydroxybenzoate under iron limitation - or as a ferrisiderophore reductase, which would divert part of its reducing potential away from chorismate synthase in the presence of iron.

Our results suggest that such a model is not operating in $S$. cerevisiae. Production of AA and OH-AA decreased 


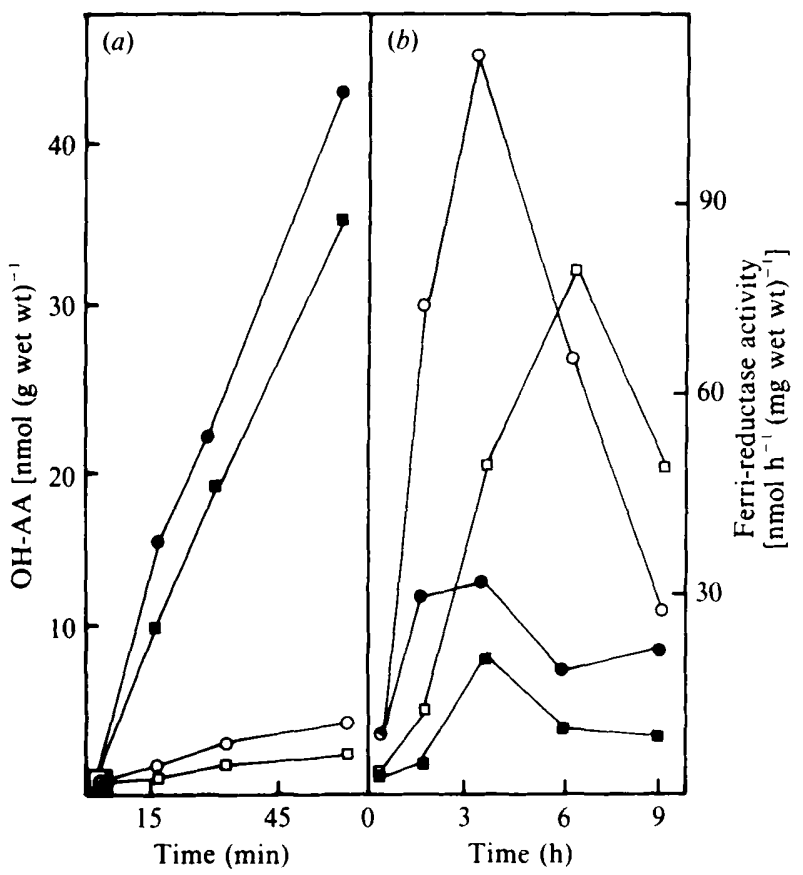

Fig. 3. Excretion of OH-AA by cells in resting conditions $(a)$ and change in the ferri-reductase activity of the cells during growth $(b)$. (a) - Strain FL200 (wild-type); O, strain G204; $\square$, strain F113 (wildtype); $\square$, strain W103. (b) O, Strain X2180-1A (wild-type), iron-rich medium; $\square$, strain RH1127, iron-rich medium; $O$, strain X2180-1A, iron-deficient medium; 9 , strain RH1127, iron-deficient medium.

under iron limitation. If there were competition for reducing equivalents between chorismate synthase and ferri-reductase, then the ferri-reductase activity in whole cells of the chorismate-synthase-deficient mutant should be greater, but this was not the case (Fig. $3 b$ ). The problem could, however, be more complicated, since there are several enzymes with ferric-iron-reducing properties associated with different subcellular fractions in $S$. cerevisiae (Lesuisse et al., 1990).

The presence of OH-AA in all wild-type strains of $S$. cerevisiae raises the question of whether the metabolism of tryptophan by yeast cells involves the tryptophan oxygenase pathway, which has not been detected so far in $S$. cerevisiae (see Gaertner, 1981) or an alternative (oxidative?) pathway starting from anthranilate.

Finally, our results suggest that in yeast there is some link between the excretion of aromatics and iron metabolism (see, for example, Fig. $3 a$ ). The nature of this link and the physiological role of excretion of aromatics by $S$. cerevisiae remain unknown, but studies are in progress in order to clarify these questions.
E. Lesuisse was the recipient of a grant from the Commission of the European Communities (Brussels, Belgium). This work was supported by grants from CNRS and Universite Paris VII. We thank Dr G.H. Braus and Dr A. Dancis for the generous gift of yeast strains and $\operatorname{Dr} O$. Parkes for help in preparing the manuscript.

\section{References}

DOWNER, D. N., DAvis, W. B. \& Byers, B. R. (1970). Repression of phenolic acid synthesizing enzymes and its relation to iron uptake in Bacillus subtilis. Journal of Bacteriology 101, 181-187.

Dancis, A., Klausner, R. D., Hinnebusch, A. G. \& Barriocanal, J. G. (1990). Genetical evidence that ferric reductase is required for iron uptake by Saccharomyces cerevisiae. Molecular and Cellular Biology 10, 2294-2301.

ESTABROOK, R. W. (1962). Fluorometric measurements of reduced pyridine nucleotides in cellular and subcellular particles. Analytical Biochemistry 4, 231-245.

Gaertner, F. A. (1981). A response to a letter from David Perkins concerning the question of whether nt mutants involve tryptophan pyrrolase. Neurospora Newsletter 28, 12.

Gaines, C. G., Lodge, J. S., Arceneaux, J. E. L. \& Byers, B. R. (1981). Ferrisiderophore reductase activity associated with an aromatic biosynthetic enzyme complex in Bacillus subtilis. Journal of Bacteriology 148, 527-533.

Hasan, N. \& Nester, E. W. (1978). Purification and characterization of NADPH-dependent flavin reductase. An enzyme required for the activation of chorismate synthase in Bacillus subtilis. Journal of Biological Chemistry 253, 4987-4992.

JONES, D. G. L. \& BRAUS, G. H. (1990). Cloning and characterization of the $A R O 2$ gene of the yeast Saccharomyces cerevisiae. Yeast 6 (Special Conference issue), S248.

Lesuisse, E. \& LABBE, P. (1989). Reductive and non-reductive mechanisms of iron assimilation by the yeast Saccharomyces cerevisiae. Journal of General Microbiology 135, 257-263.

Lesuisse, E., RAguzzi, F. \& Crichton, R. (1987). Iron uptake by the yeast Saccharomyces cerevisiae: involvement of a reduction step. Journal of General Microbiology 133, 3229-3236.

Lesuisse, E., Crichton, R. \& LABBE, P. (1990). Iron-reductases in the yeast Saccharomyces cerevisiae. Biochimica et Biophysica Acta 1038, 253-259.

Miozzari, G., Niederberger, P. \& Hutter, R. (1978). Tryptophan biosynthesis in Saccharomyces cerevisiae: control of the flux through the pathway. Journal of Bacteriology 134, 48-59.

Olsen, R. A., Clark, R. B. \& Bennett, J. H. (1981). The enhancement of soil fertility by plant roots. American Scientist 69, 378-384.

Rioux, C. R., Jordan, D. C. \& Rattray, J. B. (1986). Iron requirement of Rhizobium leguminosarum and secretion of anthranilic acid during growth on an iron-deficient medium. Archives of Biochemistry and Biophysics 248, 175-182.

ROMHELD, V. (1987). Existence of two strategies for the acquisition of iron in higher plants. In Iron Transport in Microbes, Plants and Animals, pp. 353-374. Edited by G. Winkelmann, D. Van der Helm \& J. B. Neilands. Weinheim \& New York: VCH.

Welch, G. R., KenNeth, W. C. \& Gaertner, F. H. (1974). Chorismate synthase of Neurospora crassa : a flavoprotein. Archives of Biochemistry and Biophysics 165, 505-518.

WinkelmanN, G. \& HuschKa, H.-G. (1987). Molecular recognition and transport of siderophores in fungi. In Iron Transport in Microbes, Plants and Animals, pp. 317-336. Edited by G. Winkelmann, D. Van der Helm \& J. B. Neilands. Weinheim \& New York: VCH. 